\title{
An Economic Analogy to Electrodynamics
}

\author{
Sanjay Dasari ${ }^{1}$, Anindya Kumar Biswas ${ }^{2}$ \\ ${ }^{1}$ Malad Office, Mumbai Branch, JP Morgan India Pvt. Services Ltd., India \\ ${ }^{2}$ Department of Physics, North-Eastern Hill University, Mawkynroh-Umshing, Shillong, India \\ Email: anindya@nehu.ac.in
}

Received August 21, 2013; revised September 18, 2013; accepted September 28, 2013

Copyright (C) 2013 Sanjay Dasari, Anindya Kumar Biswas. This is an open access article distributed under the Creative Commons Attribution License, which permits unrestricted use, distribution, and reproduction in any medium, provided the original work is properly cited.

\begin{abstract}
In this article, we would like to find the laws of electrodynamics in simple economic systems. We identify the chief economic variables and parameters, scalar and vector. We find laws of economics interms of these variables and parameters. The laws are similar in form to the laws of symmetric electrodynamics. Moreover, we obtain Phillp's curve, recession, Black-Scholes formula, Supply-Demand line and Cobb-Douglas production function as sample applications.
\end{abstract}

Keywords: Economics; Electrodynamics; Duality

\section{Introduction}

"We want the most but cannot have it" as a saying goes. The saying says about the problem of scarcity, talks about the problem of duality. The explicit and implicit statement of the saying is at the core of the branch of economics. Economics being a system and a problem have mesmerised many, including physicists. Physicists have tried to comprehend the complexity of economics from time immemorial, starting from Copernicus, through Isaac Newton to Eugene Stanley [1-3].

The question keeps coming, can we understand economics as simply as mechanics [4]? Can we comprehend force laws behind economic developments as simply as four force laws in physics? Though there are few interesting attempts [5-7], direct attacks to answer the questions probably are missing.

How a system changes position, in a given environment, as a function of time in the study of mechanics. How a given charged environment dictates a charged particle has been the branch of electrodynamics dealing with, with electrodynamics coming electric and magnetic fields as well as electric and magnetic charges. Though the magnetic charge is yet to be discovered, the dichotomy, or duality of electrics and magnetics is at the heart of enormous amount of theoretical constructions. One such construction is symmetric electrodynamics due to Cabibo and Ferrari [8].

In this article, we will refer to the easily available books on electrodynamics [9] and economics [10], while trying to separate, step by step, one kind of force law in action in economics. We do this in the following way. First we describe the Maxwell's equations of electrodynamics as well as continuity equation and Lorentz force law in the Section 2. Then we introduce the chief economic variables and formulate the correspondence of the economic variables to the standard electrodynamic variables and parameters in the Section 3 and Section 4 respectively. Virtues of competition were estimated highly by pioneering Adam Smith [11]. Competition flow, here, is one of the chief economic variables. In the next step, in the Section 5, we verify how equations of electrodynamics are holding good in economic systems. We also consider analogue of materials in economics in the Section 6. Potential formulation of electrodynamics is a powerful solution technique. We will see how that also descends down to us in economics in the Section 7.

Formulation of economics based on the analogy to Maxwellian electrodynamics is sufficient to account for money and scarcity. To account for capital and labour we require symmetric completion of Maxwellian electrodynamics. Capital and labour get their due places as monopole and antimonopole get in symmetric electrodynamics [8].

Unemployment, inflation of prices are day to day headache. Recession was the first word of the song for the day to start with until few years back. What is less heard is that there is an empirical graphical relation between inflation rate and unemployment rate, in the short run. The name of the line is Phillip's curve, originally 
due to an alternative form of A. W. Phillips [12]. We derive sort of Phillip's curve using the rules, describe the recession also in the Subsections 8.1 and 8.2 respectively. Moreover, option trading (one type of booking share) is something that makes the share market efficient. Pricing of the option has been a long standing academic issue. F. Black and M. Scholes were the first to, using intuition from Physics, namely diffusion equation of heat, give a reasonable formula [13] for the call (and hence put) option. In this article, we re-derive the Black-Scholes formula, visualising call option as one component of profit flow rather than temperature, as a particular case of more general class of feasible formulas in the Subsection 8.3. Unobservable factor market volatility, also gets split up. As sample applications requiring capital-labour sector, we derive supply-demand line and Cobb-Douglas production function in the Subsections 8.4 and 8.5 respectively. In Appendix of Section 8 we describe how we can embed utility in this formulation. We consider subtle points, broader outlook and conclusion in the Sections 9-11 respectively.

We will take India and Indian currency, Rupee, as a background for our purpose of the paper. But the full content will be holding true, if India and Indian currency are replaced globally, in this article, by any country and the corresponding currency.

\section{Maxwell's Equations}

We recall that the basic variables of electrodynamics are electric field, $\boldsymbol{E}$ and magnetic field, $\boldsymbol{B}$. These two fields can exist without, can generate in a medium or, can be produced by electric (magnetic) charge density, $\rho_{e}\left(\rho_{m}\right)$ and electric current density, $\boldsymbol{j}_{e}\left(\boldsymbol{j}_{m}\right)$. The relations, whenever relevant, between electromagnetic fields and charge(s) (current(s)) in a vacuum (material medium) are fixed by permittivity constant, $\epsilon_{0}\left(\epsilon_{0} \epsilon_{r}\right)$, and permeability constant, $\mu_{0}\left(\mu_{0} \mu_{r}\right)$. These six variables have an interesting interrelationship. Moreover, the charge density and current constrain each other through a constitutive relation. Let us describe along that line in the paragraph to follow.

The four equations of symmetric electrodynamics are as $[8,9]$

$$
\begin{gathered}
\epsilon_{0} \nabla \cdot \boldsymbol{E}=\rho_{e} \\
\nabla \times \boldsymbol{E}=-\frac{\partial}{\partial t} \boldsymbol{B}-\mu_{0} \boldsymbol{j}_{m} \\
\nabla \cdot \boldsymbol{B}=\mu_{0} \rho_{m} \\
\nabla \times \boldsymbol{B}=\mu_{0} \epsilon_{0} \frac{\partial}{\partial t} \boldsymbol{E}+\mu_{0} \boldsymbol{j}_{e} .
\end{gathered}
$$

The constitutive relation(s), called continuity equation(s), is

$$
\nabla \cdot \boldsymbol{j}_{e, m}+\frac{\partial}{\partial t} \rho_{e, m}=0 .
$$

The force acting on a charge (electric + magnetic) distribution is given by the Lorentz Force Law

$$
\boldsymbol{F}=\rho_{e}(\boldsymbol{E}+\boldsymbol{v} \times \boldsymbol{B})+\rho_{m}\left(\boldsymbol{B}-\frac{1}{c^{2}} \boldsymbol{v} \times \boldsymbol{E}\right) .
$$

Ala Singleton [8], we write $\boldsymbol{E}$ and $\boldsymbol{B}$ interms of two four potentials as

$$
\begin{gathered}
\boldsymbol{E}=-\nabla \phi_{e}-\frac{\partial \boldsymbol{A}}{\partial t}-\nabla \times \boldsymbol{C}, \\
\boldsymbol{B}=-\nabla \phi_{m}-\frac{\partial \boldsymbol{C}}{\partial t}+\nabla \times \boldsymbol{A} .
\end{gathered}
$$

In the Coloumb gauge, the Equations (1)-(4), reduce to

$$
\nabla^{2} \phi_{e, m}=-\rho_{e, m}
$$

and the corresponding Poisson's equations for vector potentials.

The Equations (1)-(5) are covariant whereas Equation (6) is invariant under the duality transformation

$$
\begin{gathered}
\boldsymbol{E}^{\prime}=\boldsymbol{E} \cos \alpha+c \boldsymbol{B} \sin \alpha \\
c \boldsymbol{B}^{\prime}=-\boldsymbol{E} \sin \alpha+c \boldsymbol{B} \cos \alpha \\
c \rho_{e}^{\prime}=c \rho_{e} \cos \alpha+\rho_{m} \sin \alpha \\
\rho_{m}^{\prime}=-c \rho_{e} \sin \alpha+\rho_{m} \cos \alpha \\
c \boldsymbol{j}_{e}^{\prime}=c \boldsymbol{j}_{e} \cos \alpha+\boldsymbol{j}_{m} \sin \alpha \\
\boldsymbol{j}_{m}^{\prime}=-c \boldsymbol{j}_{e} \sin \alpha+\boldsymbol{j}_{m} \cos \alpha \\
c \phi_{e}^{\prime}=c \phi_{e} \cos \alpha+\phi_{m} \sin \alpha \\
\phi_{m}^{\prime}=-c \phi_{e} \sin \alpha+\phi_{m} \cos \alpha .
\end{gathered}
$$

Moreover, $c^{2} \phi_{e}^{2}+\phi_{m}^{2}$ remains invariant under duality transformation.

\section{Analogous Economic Variables}

We denote the main economic variables as follows:

- competition flow as $\boldsymbol{c}$

- profit flow as $\boldsymbol{P}$

- money flow as $\boldsymbol{M}$

- money density, money per unit volume, as $n$

- Ambition of a person as $\mathbf{A m}$

- Price index desirable by a consumer as $P i_{c}$

- Price index desirable by a supplier as $P i_{s}$

- Choice flow of a consumer (supplier) as $\boldsymbol{C h}_{c, s}$

- Economic power flow as $\boldsymbol{E}_{p}$

- Economic activity as $E_{a}$

- inverse of basic strength-scale of currency, at least for macro economy, as $s_{0}$

- basic technical knowhow + political power, at least for macro economy, as $k_{0}$ 
- human infrastructure as $h$

- capital density as $\rho_{K}$

- capital flow as $K$

\section{Correspondence}

- $E \leftrightarrow C$

- $\boldsymbol{B} \leftrightarrow \boldsymbol{P}$

- $\boldsymbol{j}_{e} \leftrightarrow-\boldsymbol{M}$

- $\rho_{e} \leftrightarrow-n$

- $\boldsymbol{j}_{m} \leftrightarrow \boldsymbol{K}$

- $\rho_{m} \leftrightarrow \rho_{K}$

- $\epsilon_{0} \leftrightarrow S_{0}$

- $\mu_{0} \leftrightarrow k_{0}$

- $\epsilon_{0} \epsilon_{r} \leftrightarrow s$

- $\mu_{0} \mu_{r} \leftrightarrow k$

- $\sigma \leftrightarrow h$

- $v \leftrightarrow A m$

- Scalar potential, $\phi_{e, m} \leftrightarrow-P i_{c, s}$

- Vector potential, $\boldsymbol{A} \leftrightarrow-\boldsymbol{C h}_{c}$

- Vector potential, $\boldsymbol{C} \leftrightarrow{ }_{1}-\boldsymbol{C h}_{s}$

- Poynting vector, $\boldsymbol{S}=\frac{1}{\mu_{0}} \boldsymbol{E} \times \boldsymbol{B} \leftrightarrow \boldsymbol{E}_{p}$

- energy density $\leftrightarrow E_{a} \mu_{0}$

- $\sigma_{\text {cross }}$ multiplied by power, $P \leftrightarrow\langle$ employment $\rangle$, employment generation rate.

\section{Analogy Brought Inside Out}

\subsection{Maxwell's Equations}

- Excess liquidity stimulates economic activity i.e. generates competition. Faraway from mints, activity drops to zero, competition fizzles out.

To understand it better, let us consider the following simple situation, one has left a one rupee note on the road separating two parts of a market, it will lead to a competition among the onlookers to pick it up. Imagine, instead one lakh rupee note kept on the road. It will lead to fiercer competition among the onlookers. Not only that competition which is under way along the road or, along either part of the market, will get a component across the road. Hence money density in a place generates divergence in competition flow and proportional. This is proportional at least to the first approximation. Moreover, competition points towards the money.

Let us think the exactly same situation happening twenty five years back. Then, one rupee note would have given the same divergence in the competition flow as ten thousand rupees give today. Within past twenty five years, rupee has gotten devalued by huge amount. Hence, the proportionality factor $s_{0}$ stands for the inverse of strength-scale of the currency.

This sequence of arguments follow even if we consider not this kind of free notes but constrained notes. We mean, the same kind of situation will arise with the salary of an advertised job also. We will be concerned in this paper with competition associated with the constrained notes.

Hence we deduce the first law analogous to the Equation (1)

$$
s_{0} \nabla \cdot \boldsymbol{c}=-n
$$

In this sense, money density is analogue of negative charge density. Scarcity is analogue of positive charge. Scarcity density is more like hole density than free positive charge density. Note and scarcity, in equal magnitude form dipole. An arbitrary distribution of note (scarcity) over space can be cast into the form of multipole expansion.

In an organisation, when money is not flowing or, notes are stationary there is no competition. This is like $\boldsymbol{E}=0$ in a conductor.

- In general profit is a composite object composed of money, labor etc. In the simplest cases profit is quantified as money gain. In any exchange, positive profit of one is equal to, in magnitude, the negative profit of the other. Hence, in any exchange, net change in profit is zero. If there is no exchange, there is no change in profit, either way. Hence, we have

$$
\nabla \cdot \boldsymbol{P}=0 .
$$

Let's consider an isolated primitive economy. Let's recall in this context the popular phrase "hand to mouth". The profit is starting from hand and ending at mouth. If we focuss locally on hand or, mouth, the above Equation (19), is not holding true. Hand is the source for profit, mouth is the sink. So we should introduce a source term on the right hand side of the Equation (19). Hand stands for labour. Extended hand, in economic parlance, is capital. As we graduate through closed to open economy, the appropriate definition, it appears to us, for labour is the non-signalling head + torso of an animate object. Hands, feet, signalling mouth, traditional capital like tools, cabs, houses, lands, old technologies and new technologies like software, internet, nano-tech or, stem-cell therapy. etc. comprise capital. With this definition of capital and labour, these two stand for positive and negative single pole sources for profit. The part of the profit lines starting from hand and ending at nearby head+torso are wage-lines, the rest other profit-lines are ending at somewhere else and termed as profit in traditional language. Hence, the Equation (19) is a special case of a general equation

$$
\nabla \cdot \boldsymbol{P}=k_{0} \rho_{K}
$$

where, $\rho_{K}$ stands for capital density and $k_{0}$ is the basic sophistication scale of the capital. Capital is positive, labour is negative. Capital, labour can form dipole like machine and mechanic.

- Profit flow coming from retail chain sector leads local businessmen to get united and protest. Protest is a 
form of competition flow. We may note that this is what experienced in pure diamagnetic phenomenon or, when a bar magnet is pushed orthogonally towards a wire loop. Initial reactions to software coming to India were also similar. This motivates us to write

$$
\nabla \times \boldsymbol{c}=-\frac{\partial}{\partial t} \boldsymbol{P}
$$

This also indicates that Faraday's law boils down to Ricardo's principle in economics.

The Equation (21) takes the form, in the presence of capital,

$$
\nabla \times \boldsymbol{c}=-\frac{\partial}{\partial t} \boldsymbol{P}-k_{0} \boldsymbol{K} .
$$

Just recall competition of biofarms about new patents, family members about a house, companies about contracts (say, gas, band-width, oil etc.). Competition repels house to come to anyone in the family.

- Like magnetic field profit is also non-conservative field. If there is no money, there is no profit. Circulation of notes gives rise to profit. As money starts incoming more and more to a place, profit also increases, say in a place, to some people more and more. As money comes more, differences in money contents from person to person, say, increase more. Rich becomes richer, poor becomes poorer. This is a local consideration.

Let us think of the opposite limit, where there is no money flow into a place. But if competition flow, say promotional competition in a company, changes with time, like in some months of the year, this leads to more spending, hence more profit circulation in the local economy or, micro-economy. Product differentiation too leads to circulation of profit in a local economy. These considerations lead us to the relation

$$
\nabla \times \boldsymbol{P}=s_{0} k_{0} \frac{\partial}{\partial t} \boldsymbol{c}-k_{0} \boldsymbol{M} .
$$

\subsection{Continuity Equation}

We know that no one creates (destroys) money, unless one is crazy. The amount of money that enters (goes out) from one's pocket, or, from one ATM, or, from one bank, in unit time is just equal to the rate of change of money in that pocket or, ATM or, the bank. This is just the continuity Equation (5).

But there is an exception. Notes are destroyed or, generated at the $\operatorname{mint}(\mathrm{s})$, leading to appreciation or, depreciation w.r.t. a standard currency.

So the relation (5) takes the following form, in case of economics

$$
\nabla \cdot \boldsymbol{M}+\frac{\partial}{\partial t} n=\frac{\partial}{\partial t} n_{p}
$$

where, $n_{p}$ is the amount of money being printed or, destroyed in a mint.

Applying the same kind of logic, we get to a continuity equation for capital flow,

$$
\nabla \cdot \boldsymbol{K}+\frac{\partial}{\partial t} \rho_{K}=\frac{\partial}{\partial t} \rho_{K_{p}}
$$

where, $\rho_{K_{p}}$ is the density of capital being created or, destroyed.

\subsection{Lorentz Force Law}

Let us imagine, competition has started flowing in a place, buy a house or, buy sports goods or, buy a ticket for a show. A person will respond or, not and if responds to what extent, depends on how much money is there in his pocket. Whether a locality around an ATM will respond or, not or, to what extent will depend on how much notes are there at the ATM. Response varies directly also with the appeal or, magnitude of the competition flow. So the force along the competition flow on a person or, a local society around an ATM is proportional to the competition flow, to the first approximation and the proportionality factor is money density. The same thing occurs for a nation about a Federal bank, in response to an oncoming competition flow. Here, we are meaning by competition flow, social competition flow.

Let us consider an opposite situation. Reality sector boom is coming onto a place, along the "third dimension". A person will respond provided he has business ambition. The response will be proportional to the money he owes. Once he responds this will give sidewise pushes to the people around him, who might be harbouring academic ambition only, on-setting competition along the direction perpendicular to the person's ambition direction and the profit flow direction.

Hence we heuristically come down to an equation of economic force, which is exactly the same form as Lorentz force law

$$
\boldsymbol{F}=-n(\boldsymbol{c}+\boldsymbol{A m} \times \boldsymbol{P}) .
$$

Here, we observe that only competition flows cannot give a man having scarcity, equilibrium but profit flows can. This is like Earnshaw's theorem. Second part of the statement is like magnetic confinement of charge. The same kind of logic in presence of capital leads us to

$$
\boldsymbol{F}=-n(\boldsymbol{c}+\boldsymbol{A} \boldsymbol{m} \times \boldsymbol{P})+\rho_{K}\left(\boldsymbol{P}-\frac{1}{c^{2}} \boldsymbol{A} \boldsymbol{m} \times \boldsymbol{c}\right) .
$$

Interestingly, the two terms with opposite sign, is the potential source of oscillation for any economy. In macroeconomy, we are familiar with observed business cycles.

Here, we also notice that two twins having the same money, same ambition and subjected to the same compe- 
tition and profit flows, will feel the same force. But depending on their accumulated entrepreneul skills their venture accelerations will be different. For example, one will set-up a cyber cafe much earlier than other, if the first one has software and little bit management training whereas the second one does not have that skill set. Hence economic inertial mass of a person is reciprocal of the number of his entrepreneul skills. We denote from hereon,

- economic inertial mass $=M_{e}$

- $\quad$ Number of skills $=N_{\text {es }}$

The same story will follow for two twin companies or, two twin countries. Hence we have the following identification

- $M_{e}=\frac{1}{N_{e s}}$

In the next section, we will discuss analogue of material and conductivity, restricting ourselves to the electric charge sector.

\section{Material}

Let us think that competition flow is oncoming to a place. This will create money accumulation among some and scarcity among others, giving rise to something like polarisation, bound money density at the surface of the society and at the volume. As a consequence, net competition flow will be different from the external competition flow. For weakly responsive society, polarisation vector will be equal to $s_{0} R_{c} c . R_{c}$ is the measure of the response of the society. $\boldsymbol{c}$ refers to the net competition flow in the society. The Equation (18) will get modified to

$$
\nabla \cdot s c=-n .
$$

$n$ refers to external money density.

$$
s=s_{r} s_{0}=s_{0}\left(1+R_{c}\right) .
$$

Similarly, profit flow leads to bound surface and volume circulation of notes. This results in the net profit flow differing from the external profit flow vector. This leads to a relation modified from the Equation (19)

$$
\nabla \cdot k \boldsymbol{P}=0
$$

where,

$$
k=k_{0} k_{r}=k_{0}\left(1+R_{p}\right) .
$$

Probably, $s, k$ span a two dimensional plane. Presumably, existence of black market is an example of $s, k$ being both negative [14].

Profit and competition flows both polarize.

Sometimes economy is conducive. Competition vector is proportional to money flow vector or, liquidity just like in conductor,

$$
\boldsymbol{j}=\sigma \boldsymbol{E}
$$

Proportionality factor, $h$, in economic system, like conductivity, is a measure of the quality of the human infrastructure of the company. So we have here the following rule

$$
\boldsymbol{M}=-h \boldsymbol{c} .
$$

In highly efficient $(h \rightarrow \infty)$ organisation, internal competition is zero always, which is like in metal $(\sigma \rightarrow \infty)$. h can stand for HumanCapital [15].

\section{Potential Formulation}

To show the form of the scalar potential, let us notice the following,

$$
\boldsymbol{c}=-\nabla\left(-P i_{c}\right), \boldsymbol{P}=-\nabla\left(-P i_{s}\right)
$$

implies

$$
\nabla^{2} P i_{c}=-n \frac{1}{s_{0}}, \nabla^{2} P i_{s}=+k_{0} \rho_{K} .
$$

As money density increases, Price index also increases, we see inflation.

Price index over space and time is determined by two considerations

- Prices and consumption ratios of various items at a place at a given time.

- Prices and consumption ratios of items at another time and/or at another place, compared to the base prices and consumption ratios.

The prices and consumption ratios of items change continuously over the space and time.

Hence, Price index, $P i_{c, s}$, change continuously over space and time. So, Price index, $P i_{c, s}$, is analogous to scalar potential, $\phi_{e, m}$. The first consideration sets a fixed reference value to the Price index for all other places at that time as well as for all other times. A relevant fact worth mentioning in this context is that gas index in US is based on the price of gas at a point where majority of the gas pipelines intersect.

To show the form of the vector potential, let us notice the following,

$$
\nabla^{2} \boldsymbol{C h}_{c}=-k_{0} \boldsymbol{M}, \nabla^{2} \boldsymbol{C} \boldsymbol{h}_{s}=k_{0} \boldsymbol{K}
$$

wherever, choice flow is divergence less. This continues to be as long as there is no will.

Hence, $\boldsymbol{C h}_{c}$ is in the same direction as $\boldsymbol{M}$, as $\boldsymbol{C} \boldsymbol{h}_{s}$ is in the opposite direction as that of $K$ which is our experience.

Moreover, $\left(P i_{c, s}, \boldsymbol{C h}_{c, s}\right)$ can be combined into a four vector. Ambition, Am , multiplied by Price index can be choice. Maximum Ambition is determined by the velocity of light and in fact, may be taken as velocity of light. We would like to move in any direction with the magnitude of velocity of light, c, given chance. Therefore 
it's quite plausible to write

$$
\boldsymbol{C h}^{\prime}=\frac{\boldsymbol{C h}-\boldsymbol{A} \boldsymbol{m} P \boldsymbol{i}}{\sqrt{1-\frac{A m^{2}}{c^{2}}}} .
$$

The Equations (18), (20), (22) and (23) are covariant whereas Equation (27) is invariant under the duality transformation

$$
\begin{gathered}
\boldsymbol{c}^{\prime}=\boldsymbol{c} \cos \alpha+c \boldsymbol{P} \sin \alpha, \\
c \boldsymbol{P}^{\prime}=-\boldsymbol{c} \sin \alpha+c \boldsymbol{P} \cos \alpha, \\
-c n^{\prime}=-c n \cos \alpha+\rho_{K} \sin \alpha, \\
\rho_{K}^{\prime}=c n \sin \alpha+\rho_{K} \cos \alpha, \\
-c \boldsymbol{M}^{\prime}=-c \boldsymbol{M} \cos \alpha+\boldsymbol{K} \sin \alpha, \\
\boldsymbol{K}^{\prime}=c \boldsymbol{M} \sin \alpha+\boldsymbol{K} \cos \alpha, \\
c P i_{c}^{\prime}=c P i_{c} \cos \alpha+P i_{s} \sin \alpha, \\
P i_{s}^{\prime}=-c P i_{c} \sin \alpha+P i_{s} \cos \alpha .
\end{gathered}
$$

Moreover, $\mathrm{c}^{2} \mathrm{Pi}_{c}^{2}+\mathrm{Pi}_{s}^{2}$ remains invariant under duality transformation.

\section{Application}

In the next three subsections we restrict ourselves to the electric charge sector solely.

\subsection{Phillip's Curve}

We know, in economics, Inflation rate, $\Pi$ is defined as

$$
\Pi=\frac{\mathrm{d}}{\mathrm{d} t} \ln P i .
$$

Since,

$$
\begin{gathered}
\phi_{e} \leftrightarrow P i_{c}, \\
\frac{\mathrm{d}}{\mathrm{d} t} \ln \phi_{e} \leftrightarrow \Pi
\end{gathered}
$$

or, time derivative of logarithm of scalar potential is expected to show features of economic inflation. To proceed along that line, we note from the theory of radiation in electrodynamics,

$$
\frac{\mathrm{d}}{\mathrm{d} t} \ln \phi_{e}=\omega,
$$

for electric dipole radiation, whereas, the total power radiated by the dipole is given by

$$
\langle P\rangle=\operatorname{constan} t \omega^{4} \text {. }
$$

Hence,

$$
\frac{\mathrm{d}}{\mathrm{d} t} \ln \phi_{e} \sim\langle P\rangle^{\frac{1}{4}}
$$

Here we recall that when an electromagnetic radiation falls on a medium, three processes occur. For low energy, photoelectric effect is the dominant process. As the energy increases of the infalling radiation, Compton scattering starts becoming important. At still higher energy, pair production takes over. For the photoelectric effect, cross-section, $\sigma_{\text {cross }}$, or, probability for the process to occur

$$
\sigma_{\text {cross }} \sim \frac{1}{\omega^{\frac{7}{2}}} .
$$

Photoelectric effect is producing free electrons at the cost of work-function. This phenomenon is exactly similar to employment generation from the pool of unemployed youth at the cost of lump sum money. In India, this is like giving one-time small money/loan to buy say an auto/a cab to an unemployed young man and making him self-employed. Hence, employment generation rate, denoted as $\langle$ employment $\rangle$ is the analogue of total transition rate, $\langle P\rangle \sigma_{\text {cross }}$. But

$$
\langle P\rangle \sigma_{\text {cross }} \sim \omega^{\frac{1}{2}} .
$$

Or,

$$
\langle\text { employment }\rangle \leftrightarrow \omega^{\frac{1}{2}} .
$$

Again we know, product of employment generation rate and unemployment generation rate is constant, because the two processes occur in mutually exclusive sectors, influencing each other in extreme cases, viz. percolation of software jobs to mechanical and clerical sectors. In other words,

$$
\langle\text { employment }\rangle\langle\text { unemployment }\rangle=\text { constant } .
$$

This implies

$$
\begin{gathered}
\langle\text { unemployment }\rangle \leftrightarrow \frac{1}{\omega^{\frac{1}{2}}}, \\
\langle\text { unemployment }\rangle^{-2} \leftrightarrow \omega .
\end{gathered}
$$

At the same time, Equations (45) and (46), together mean for the Inflation rate, $\Pi$

$$
\Pi \leftrightarrow \omega .
$$

Since two economic quantities, $\Pi$ and

$$
\langle\text { unemployment }\rangle^{-2} \text {, }
$$

are analogue of $\omega$, these two must be proportional to each other. In other words, for the low scale economic activity inflow,

$$
\Pi \sim \frac{1}{\langle\text { unemployment }\rangle^{2}} .
$$


This is nothing but Phillip's curve, qualitatively. Moreover, we note that the ongoing analysis in this section is reminiscent of finding relation between variables in physics, using dimensional analysis.

On the other hand, Compton scattering is pumping money in risky assets. Pair production is like bringing an woman to work place at the cost of a vacancy at the household cores. As a result, in the domain where Compton scattering becomes important [16],

$$
\sigma_{\text {cross }} \sim \frac{1}{\omega} \ln \omega .
$$

Then

$$
\Pi \sim \frac{1}{\langle\text { unemployment }\rangle^{\frac{1}{3}}} .
$$

apart from the slowly varying scale-dependent logarithmic part.

Hence, in the scale of economic activity inflow, $\left|\boldsymbol{E}_{p}\right|$ where, Compton scattering-type of phenomenon becomes important compared to photoelectric type, we get sudden increase of inflation with unemployment. This is stagflation. This is stagflation with scale-dependence setting in. If one is interested in total absorption crosssection, one can look in [17] as well as in [18] and surmise about the details of the ensuing Inflation vs unemployment curve.

For small range of time, we get one kind of society, say one kind of regime, throughout the world. This is like one kind of material, say lead, throughout the whole space. For this situation, if we consider inflation rate in the horizontal direction and unemployment rate in the vertical direction, for the same dipole, for one " $\omega$ " range, say in $\Delta \omega$, for $\Delta$ inflation rate we get one kind of $\Delta$ unemployment rate. But let us take many regimes, (long range), or, correspondingly many materials, say copper, zinc, lead etc. For those for a given $\Delta$ inflation rate we will have a series of $\Delta$ unemployment rates, many nonsmooth, say having discrete transitions from photoelectric to Compton . As a result, weighted sum of $\Delta$ unemployment rates in one $\Delta$ inflation rate will not join, in general, with that of the neighbouring $\Delta$ inflation rate. Consequently, we do not get a smooth curve in the long range. In other words, the empirical relation, Phillip's curve, exists only for short run.

\subsection{Recession}

A Recessing phase corresponds to one inertial frame for a macro-economy. The recessing inertial frame has lower ambition, $\left|\boldsymbol{A}_{m}\right|$, with respect to that of an almost contemporary macro-economy. Going to the recessing frame occurs due to saturations of collective biological activities of the society attached with the macro-eco- nomy.

The inertial frame's ambition corresponding to the macro-economy, can be thought as group ambition of the society.

As a result we see in the recessing phase, lower Price index, lower choice flow, hence lower consumption. This gets manifest through deflation, unemployment.

Since $\nabla \cdot \boldsymbol{C h}$ is not Lorentz invariant, $\nabla \cdot \boldsymbol{C h} \neq 0$ in the recessing phase. This is like at mint $\nabla \cdot \boldsymbol{M} \neq 0$. That implies number of choice lines striking a populace from one side is less than the number of lines leaving the populace in the other side. That means human will is setting in and populace is not spending to the brim. That is change in consumption pattern of commodities as well as that of prices at each place with time. This in turn will lead to lesser and lesser production and more and more unemployment.

\subsection{Black-Scholes Formula}

Let us suppose that we have gone to the stock-market armed with the set of equations we have heuristically gotten and embark on analysing the share trading. Moreover, let us focus on profit attached with call option. Then the instantaneous profit is call option value for someone having a share and writing a call option for that share. Now let us try to find the value. Let us guide ourselves by the thread of physical considerations of Black and Scholes as appears in the first few pages of the reference [13].

As long as $\boldsymbol{E}$ which is analogue of competition flow, $n$, is constant or, slowly changing with time, Maxwell's last two equations with the Ohm's law yields

$$
\nabla^{2} \boldsymbol{B}=\mu_{0} \sigma \frac{\partial \boldsymbol{B}}{\partial t} .
$$

In terms of dimensionless length variables, this Equation (59) appears as

$$
\frac{\partial \boldsymbol{B}}{\partial t}=\mu_{0} \sigma v^{2} \nabla^{2} \boldsymbol{B},
$$

where, $|v|$ is the drift speed in the medium. Translating to economic system by our dictionary and restricting us to the variation of $P$ along the third dimension, $x$, say in the stock market, we get

$$
\frac{\partial P_{i}(x, t)}{\partial t}=k_{0} h|\boldsymbol{A m}|^{2} \frac{\partial^{2} P_{i}(x, t)}{\partial x^{2}}
$$

where, for $i=1,2,3, P_{i}$ means $P_{x}, P_{y}, P_{z}$. Writing, $\tau=T-t$ and further doing the identification

- implied volatility, $\sigma=\sqrt{2 k_{0} h}|A \boldsymbol{m}|$

- $P_{i}=C(S, t) \mathrm{e}^{r \tau}=u$ is the profit at time $\mathrm{T}$, corresponding to option trading at time $t$. $C(S, t)$ is the value of the option when it is traded at time $t$. 


$$
C(S, T)=\max (S-K, 0) .
$$

We get from the Equation (61) Black-Scholes differential equation as given in the reference[19],

$$
\frac{\partial u(x, \tau)}{\partial \tau}=\frac{\sigma^{2}}{2} \frac{\partial^{2} u(x, \tau)}{\partial x^{2}} .
$$

At this point let us do some more dimensional considerations: in Option trading, relevant independent variables are

- Current stock price at time $t=S$

- Strike price or, agreed upon price of the stock at the expiry i.e. at time $T$ is $K$

- Risk less interest rate is $r$ (per year)

- Implied volatility in the stock price at time $T$ is $\sigma$ where, $\sigma^{2}$ has the dimension of time inverse (per year).

One way to combine these variables to get a dimensionless variable $x$ is to write

$$
x=\ln \frac{S}{K}+\left(r-\frac{\sigma^{2}}{2}\right) \tau .
$$

Once this is done, the straightforward solution of the Equation (62) yields the price of the call option [13,19],

$$
C(S, t)=S N\left(d_{1}\right)-K \mathrm{e}^{-r(T-t)} N\left(d_{2}\right)
$$

where,

$$
\begin{gathered}
d_{1}=\frac{\ln \left(\frac{S}{K}\right)+\left(r+\frac{\sigma^{2}}{2}\right)(T-t)}{\sigma \sqrt{T-t}}, \\
d_{2}=d_{1}-\sigma \sqrt{T-t}, \\
N(d)=\frac{1}{2 \pi \sigma^{2}} \int_{\infty}^{d} \mathrm{~d} x \mathrm{e}^{-\frac{x^{2}}{2 \sigma^{2}}} .
\end{gathered}
$$

\subsection{Supply-Demand Line}

Roughly, price index, $P i_{c}$, desirable by a consumer, is given for two commodities system as

$$
P i_{c}=100\left(\frac{x}{X} \cdot \frac{y}{y+q}+\frac{p}{P} \cdot \frac{q}{y+q}\right)=100 \frac{\frac{x}{X} y+\frac{p}{P} q}{y+q}
$$

where, prices of two commodities are $x$ and $p$; whereas the quantities are $y$ and $q$ respectively. Prices of two commodities are $X$ and $P$ in the base year.

Let us now take resort to Citrus Paribus condition i.e. assume that the price and the consumption of the second commodity remain fixed. Moreover, let's also assume that the note density and the strength scale of currency, $s$, remaining the same all over the space keeping Price index unchanging, courtsey Equation (28). Denoting the fixed Price index by $a$, we derive from Equation (64),

$$
x=X \frac{a}{100}+X \frac{q\left(\frac{a}{100}-\frac{p}{P}\right)}{y} .
$$

So the plot between the price, $x$ and the quantity of the commodity number one, $y$, is a hyperbola. We know, the middle part of the hyperbola is a straight line approximately. Hence, we have derived the equilibrium demand curve. By varying $a$, we get the series of demand lines.

The object under bargain is duality invariant. The addition of squares of price indices as desirable by consumer and supplier is constant. Hence, for a price-value of an object, addition of the squares of the quantity desirable for consumer and supplier is constant. Hence, supply-line has opposite orientation with respect to the demand-line. These two lines intersect at a point, called self-dual point. The price function, price per unit quantity, or, the supply-demand line has the shape of simple harmonic oscillator potential function. The self-dual point is the minimum of the potential function. Moreover, at the self-dual point the market clears. The self-dual point is where both the price indices agree and any one of these Price index at that point is the familiar Price index. On the other hand, the shape of the price function immediately entails possibilty of an oscillation(s) in the price, in the long run.

\subsection{Cobb-Douglas Production Function}

Production is attaching life to a substrate called material. Capital and labour combine to give life to the substrate to make it product. This is sort of reaction in which

- capital life-time lost + labour life-time lost $\rightarrow$ product life-time

This looks like

- $\alpha$ (unit capital life-time lost) $+\beta$ (unit labour life-time lost) $\rightarrow$ product life-time

Moreover, this appears like

- $\alpha A+\beta B \rightarrow C$

Now, the rate of production, $r$, is given by chemical kinetics as

$$
r=A^{\alpha} B^{\beta},
$$

where, for a complicated multi-step process, stochiometric coefficients $\alpha, \beta$, can be any positive rational number. Chemical kinetics is nothing but electromagnetic forces in action. Using our dictionary and presence of duality symmetry implies that the rate of production, $q$, will have the form

$$
q=K^{\alpha} L^{\beta} .
$$

This is the Cobb-Douglas production function [20]. 


\section{Points}

Here we touch on some delicate issues. Competition flow in this letter is separate from "pure arbitrage flow" just like profit is more than money gain. We can think of three dimensional vector spaces, locally composed of two dimensional plane and a "third dimension". For a company, the "third dimension" is hierarchy. In the stock market, the "third dimension" is the "share" direction as we have explained in the previous subsection. Normally, the "third dimension" is the third dimension, communication is being made along that electrically or, electromagnetically i.e. by land line or, satellite.

Though appear distinct, profit flow and competition flow are close by in our formulation. Profit making itself has a competitive spree within. Profit flow can be easily seen as a flow and can be measured. From the profit flow, one can, in principle, then calculate competition flow using relativistic transformations [9], for many cases. In cetain other situations, one can measure competition flow by measuring the difference of Price index as desirable by a consumer, $P i_{c}$, at two places and dividing by the distance between the two places.

Moreover, any subject has three aspects, theoretical, descriptive and applied. Our analysis is theoretical unlike many other approaches to economics from physics which are applied in nature. Our analysis is local. It has the possibility of treating spatial inflation as well as spatiotemporal inflation, in particular and spatio-temporal economic phenomena, in general.

\section{Outlook}

Naively, one tends to wonder whether the topological considerations in mathematical economics can be related to magnetic topologies. Similarly many topics in economics, elementary as well as advanced are expected to be described by electrodynamics using the dictionary introduced in this paper. One can take a straightforward route also. Consider the Equations (18), (20), (22) and (23) as the rules of economics, measure the variables and the parameters discussed, say, knowing HumanCapital ala economists, one can try to measure competition flow using Equation (31), and therefrom try to explain as many economic empirical relations as possible.

\section{Conclusion}

We have given an alternative formulation of economics. The rules of the formulation are Equations (18), (20), (22) and (23). The variables are as mentioned, e.g. profit flow, competition flow, money flow, constrained note density, capital etc. These rules are analogue of symmetric Maxwell's equations. Moreover, we have obtained continuity equation, force rule, inertial mass for an economic system and an operational definition of HumanCapital. We have constructed a 4-potential formulation. Using the model we get Phillip's curve, describe stagflation, recession. Dwelling on stock-market we recover call option function. We have gotten a scenario where, unobservable market volatility can be made observable if we can measure the drift ambition of sort-sellers. We have made the known duality in economics more rigorous. Here, note and capital are dual to each other, the supply-demand line emerges as the dual aspect of the same line, something as simple as Cobb-Douglas production function also gets a heuristic derivation via duality. Besides, we have pointed to few avenues, amidst many, along which this approach can be explored further.

\section{Acknowledgements}

To the best of our knowledge, the material covered in this manuscript was not dealt with anywhere else. We would like to thank many people for discussions, comments and suggestions. A preliminary version of this work has appeared in the arxiv, [21].

\section{REFERENCES}

[1] E. Stanley, "The Back Page in APS News 17[11]," 2008, pp. 8-9.

[2] R. N. Mantegna and H. E. Stanley, "Introduction to Econophysics: Correlations and Complexity in Finance," Cambridge University Press, Cambridge, 2000.

[3] W. M. Saslow, "An Economic Analogy to Thermodynamics," American Journal of Physics, Vol. 67, No. 12, 1999, p. 1239. http://dx.doi.org/10.1119/1.19110

[4] A. G. Pikler, "Utility Theories in Field Physics and Mathematical Economics (II)," The British Journal for the Philosophy of Science, Vol. 5, No. 20, pp. 303-318.

[5] K. N. Llinski, "Gauge Physics of Finance: Simple Introduction," 1998. http://in.arxiv.org/abs/condmat/9811197

[6] K. N. Llinski and A. S. Stepanenko, "Electrodynamical Model of Quasi-Efficient Financial Market," 1998. http://xxx.lanl.gov/abs/condmat/9806138

[7] N. Dunbar, "Market Forces," New Scientist, Vol. 2128, 1998, pp. 42-45

[8] N. Cabibo and E. Ferari, "Quantum Electrodynamics with Dirac Monopoles," Nuovo Cimento, Vol. 23, No. 6, 1962, pp. 1147-1154; D. Singleton, "Electrodynamics with Magnetic Charge and Two Photons," American Journal of Physics, Vol. 64, No. 4, April 19.

[9] D. J. Griffiths, "Introduction to Electrodynamics," 3rd Edition, Prentice-Hall, Inc., New Jersey, 1999.

[10] K. Case, R. Fair and S. Oster, "Principles of Economics," 9th Edition, Pearson Books.com, 2009.

[11] A. Smith, "Wealth of Nations," Introduced by Alan B. Krueger, Bantam Classics, Mass Market Paperback.

[12] A. W. Phillips, "The Relationship between Unemployment and the Rate of Change of Money Wages in the United Kingdom 1861-1957," Economica, Vol. 25, No. 
100, 1958, pp. 283-299.

[13] F. Black and M. Scholes, "The Pricing of Options and Corporate Liabilities," The Journal of Political Economy, Vol. 81, No. 3, 1973, pp. 637-654. http://dx.doi.org/10.1086/260062

[14] V. G. Veselago "The Electrodynamics of Substances with Simultaneously Negative Values $\epsilon$ of and $\mu$ " Soviet Physics Uspekhi, Vol. 10, No. 4, 1968, 509014. http://dx.doi.org/10.1070/PU1968v010n04ABEH003699

[15] M. P. Todaro, S. C. Smith, "Economic Development," 8th Edition, Pearson Education, Chapter 9.

[16] J. D. Bjorken and S. D. Drell, "Relativistic Quantum Mechanics," McGraw-Hill Book Compnay, Inc., 1964, p.

\section{Appendix}

\subsection{Utility}

Here, to elucidate the concept of marginal utility, let us recall the prototype example of three oranges eaten one after another, thereby reducing the demand for a consecutive orange.

Marginal utility $(M U)$ is price. Hence, total utility $(T U)$, is price index, magnitude-wise, once these are measured with money.

$$
M U=\frac{\mathrm{d} T U}{\mathrm{~d} Q}
$$

But marginal utility and price are not identical. One is subjective and another one is objective. Hence, we go one step further and define marginal utility as $i$ times price, total utility as $i$ times price-index. Therefore, square of choice flow plus square of total utility is constant, as ambition changes.

$$
\boldsymbol{C h}^{2}+T U^{2}=\text { constant }
$$

Consequently, total utility is maximumwhen the choice flow has magnitude zero. Keeping these in mind, let's analyse the example of oranges (but this time many
132.

[17] I. Kaplan, "Nuclear Physics," 2nd Edition, AddisonWesley Publishing Company, Inc., USA, 1962, 416-417.

[18] W. Heitler, "The Quantum Theory Of Radiation," 3rd Edition, Oxford University Press, London, 1954.

[19] http://en.wikipedia.org/wiki/Black-Scholes

[20] C. W. Cobb and P. H. Douglas, "A Theory of Production," American Economic Review, Vol. 18, Suppl., 1928, pp. 139-165.

[21] S. Dasari and A. K. Biswas, "An Economic analogy to Electrodynamics," 2013. http://xxx.lanl.gov/abs/physics/1001.1847

oranges). Initially, choice is in the direction of eating orange and of large magnitude. As one by one, orange is being eaten, choice flow vector gets reduced in length, as well as rotated. At one point, "no more, please!", choice becomes zero or, length of choice flow vector becomes zero. After that if one is pushed to eat she/he starts feeling nauseatic, choice flow vector has reversed its direction and increasing its magnitude with each additional orange being swallowed. So with respect to the maximum value of total utility at a particular number of oranges $\left(Q_{m}\right.$, say), (or, food set), the total utility reduces in both side as we increase or, decrease the number from $Q_{m}$. This is the standard total utility versus quantity graph found in economics book.

\subsection{Basic Equations of Economics for Quick Reference}

$$
\begin{aligned}
& s_{0} \nabla \cdot \boldsymbol{c}=-n ; \quad \nabla \cdot \boldsymbol{P}=k_{0} \rho_{k} ; \quad \nabla \times \boldsymbol{c}=-\frac{\partial}{\partial t} \boldsymbol{P}-k_{0} \boldsymbol{K} ; \quad \nabla \times \boldsymbol{P}=s_{0} k_{0} \frac{\partial}{\partial t} \boldsymbol{c}-k_{0} \boldsymbol{M} \\
& \nabla^{2} P i_{c}=-n \frac{1}{s_{0}} ; \nabla^{2} P i_{s}=+k_{0} \rho_{K} ; \quad \nabla^{2} \boldsymbol{C} \boldsymbol{h}_{c}=-k_{0} \boldsymbol{M} ; \quad \nabla^{2} \overrightarrow{\boldsymbol{C h}}_{c}=-k_{0} \vec{K}
\end{aligned}
$$

